\section{Efficient Faraday Rotation in Birefringent Optical Microfiber Loop Resonators for Current Sensing}

\author{
G. Y. Chen, G. Brambilla and T. P. Newson
}

The optimization of resonantly enhanced Faraday rotation in microfiber loop resonators with linear birefringence is presented. For a sufficiently large birefringence-induced resonance separation, the evolution of differential phase between the two orthogonal polarizations can lead to efficient Faraday rotation when the loop circumference is a quarter of the polarization beat length and the roundtrip phase of the eigenmode in the fast axis is $3 \pi / 2$ plus an integer multiple of $2 \pi$. This study provides the groundwork for fabricating microfiber loop resonator based current sensors that can operate efficiently despite the presence of birefringence.

Introduction: The dielectric nature of optical current sensors enables them to perform measurements in high voltage or magnetic fields due to their immunity from saturation effects that would otherwise limit conventional current sensors. Optical microfiber (OM) based current sensors [1-6] have gathered considerable interest due to their compact size and high bandwidth. Their sensing mechanism is either based on the Faraday Effect $[1,2]$ or thermally induced wavelength shifts [3-6]. Faraday-based measurements are less vulnerable to changes in the ambient environment, but they are highly susceptible to linear birefringence arising from micro-bending and polymer-packaging. Linear birefringence can drastically reduce the measured Faraday rotation due to the reversal in polarity every $\pi$ interval in differential phase $(\Delta \varphi)$, as shown in Fig. 1. For non-resonant sensors, this tends to restrict the maximum usable fiber length to a quarter of the polarization beat length $\left(B_{L}\right)$ if the initial $\Delta \varphi$ of the sensor head was equal to zero or integer multiples of $2 \pi[1,2]$. To extend the maximum usable fiber length to $B_{L} / 2$, the initial $\Delta \varphi$ bias must equal $\pi / 2$ or $3 \pi / 2$, plus integer multiples of $2 \pi$. This can be implemented in practice using a birefringent modulator or by selecting the appropriate fiber length, provided the birefringence is known. In this Letter, we propose design optimizations to overcome the optical path length limitation in nonideal microfiber loop resonators (MLR), resulting in efficient resonantly enhanced Faraday rotation despite the effects of linear birefringence.

Background: A MLR [7] can be fabricated from the uniform waist of a biconical fiber taper by forming a loop with self-coupling in the region of close contact. The maximum Faraday rotation for a roundtrip of the MLR (Fig. 2) can be expressed in terms of the magnetic permeability of free-space $\left(\mu_{0}\right)$, the relative permeability of silica $\left(\mu_{r}\right)$, the Verdet constant of silica $(V)$, and the current flowing through the wire $(I)$ :

$$
\theta_{\max }=\mu_{0} \mu_{r} V I
$$

As a current sensor, it is desirable for the Faraday Effect to occur at the resonant wavelength $\left(\lambda_{\text {res }}\right)$, where light confinement is at a maximum and its optical path length is considerably longer than the physical length of the loop $\left(L_{c}\right)$. The MLRs discussed in this work are assumed to be single-mode, which can be achieved in practice by limiting the $\mathrm{OM}$ diameter or ensuring an adiabatic taper transition from single-mode fiber. The initial $\Delta \varphi$ of the MLRs is assumed to be zero.

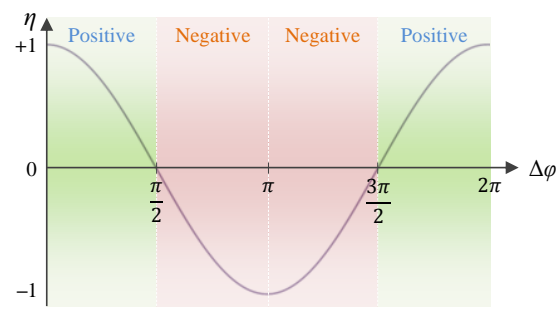

Fig. 1 Dependence of the normalized Faraday rotation $\left(\eta=\theta / \theta_{\max }\right)$ on the differential phase $(\Delta \varphi)$ between the orthogonal polarizations.

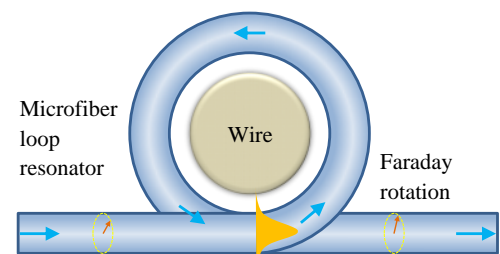

Fig. 2 Schematic of a MLR wrapped around a current-carrying wire. The current-induced magnetic field parallel to the direction of propagation of light induces a rotation in the state of polarization.

Discussions: For the ideal case in which the birefringence is zero (Fig. $3 a)$, the two eigenmodes of the orthogonal polarizations propagate with equal phase velocities and thus the Faraday rotation is at the maximum efficiency between the eigenmodes with the same propagation distance. However, the light at any point in the MLR is actually composed of eigenmodes that have entered the resonator at different times. Therefore, the maximum efficiency of Faraday rotation between the eigenmodes of different roundtrips can only be maintained if the accumulated phase $(\varphi)$ per roundtrip including the $\pi / 2$ contribution from coupling is equal to an integer multiple of $2 \pi$, as evident from the roundtrip phase analysis shown in Fig. 4a. This $\varphi$ can be translated into a set of values for $L_{c}$ in terms of $\lambda_{\text {res }}$, the effective index of the eigenmodes $\left(n_{\text {eff }}\right)$ and an integer $C$ :

$$
\begin{aligned}
& \varphi=2 \pi C=n_{\text {eff }} L_{c}\left(2 \pi / \lambda_{\text {res }}\right)+\pi / 2 \\
& L_{c}=\lambda_{\text {res }}(C-1 / 4) / n_{\text {eff }}
\end{aligned}
$$

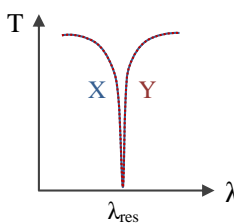

a

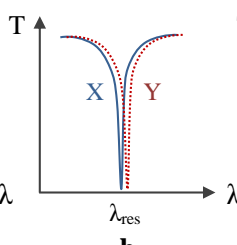

b

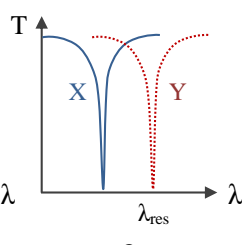

c
Fig. 3 Transmission spectra of MLRs, with: a. zero birefringence

b. small/moderate birefringence-induced resonance separation

$c$. large birefringence-induced resonance separation
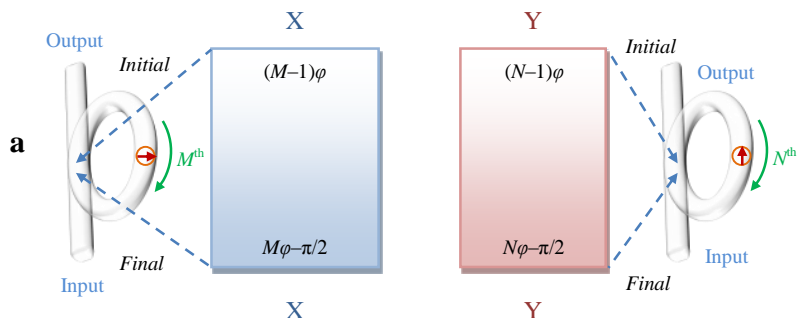

Y

b
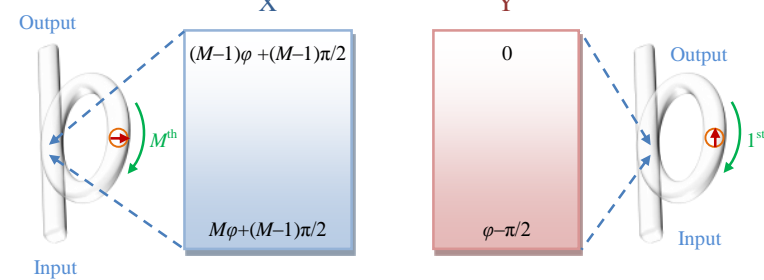

Fig. 4 Roundtrip phase analysis for MLRs operating at $\lambda_{\text {res, }}$ with: a. zero birefringence

b. large birefringence-induced resonance separation (with $B_{L}=4 L_{c}$ ) The initial (coupled in light) and final phase (before coupling back in) is labelled inside rectangles that represent the $M^{\text {th }}$ and $N^{\text {th }}$ roundtrip of the eigenmode in the slow $(X)$ and fast $(Y)$ axes respectively. 
In practice, linear birefringence exists as a result of bend- and packaging-induced stresses in the MLR. In such cases, the resonant wavelengths of the two eigenmodes are no longer identical due to different effective indices. Fig. $3 \mathrm{~b}$ and Fig. 3c illustrates the spectralsplitting phenomenon. This can be problematic for current sensing, as Faraday rotation starts in the usual manner transferring power from one axis to another, but as $\Delta \varphi$ between the eigenmodes increase beyond $\pi / 2$, the direction of Faraday rotation gradually reverses until a total cancellation at the propagation distance of half a beat length.

For MLRs with a small to moderate birefringence-induced resonance separation (Fig. 3b), there is no mutual agreement in the requirement of $\varphi$ between the eigenmodes of arbitrary roundtrips. Hence, no condition exists for efficient Faraday rotation.

For MLRs with a large birefringence-induced resonance separation (Fig. 3c) such that, at a particular wavelength one eigenmode (e.g. Xaxis) experiences maximum recirculation while the other eigenmode (e.g. Y-axis) is uncoupled. The removal of the accumulative birefringence-induced phase component in one axis simplifies the phase matching conditions and efficient Faraday rotation between the eigenmodes of arbitrary roundtrips can be obtained with $B_{L}=4 L_{c}$ and $\varphi$ (including $\pi / 2$ from coupling and excluding $\pi / 2$ from birefringence) equal to $3 \pi / 2$ plus an integer multiple of $2 \pi$. As shown in Fig. $4 b, \Delta \varphi$ only grows from 0 to $\pi / 2$ during each roundtrip. Hence, Faraday rotation is uni-directional and accumulates with the optical path length. Similarly, this $\varphi$ can be converted into a set of values for $L_{c}$ :

$$
\begin{aligned}
& \varphi=3 \pi / 2+2 \pi C=n_{\text {eff }} L_{c}\left(2 \pi / \lambda_{\text {res }}\right)+\pi / 2 \\
& L_{c}=\lambda_{\text {res }}(C+1 / 2) / n_{\text {eff }}
\end{aligned}
$$

where $n_{\text {eff }}$ is the effective index of the eigenmode in the fast axis (Y). The resonance separation $\Delta \lambda_{\text {res }}$ is related to $\lambda_{\text {res }}$, the index difference $\left(\Delta n_{e f f}\right)$ between the eigenmodes in the slow and fast axes, and $n_{\text {eff: }}$ :

$$
\Delta \lambda_{\text {res }} / \lambda_{\text {res }}=\Delta n_{\text {eff }} / n_{\text {eff }}
$$

$B_{L}$ can be expressed in terms of $\lambda_{\text {res }}$ and $\Delta n_{\text {eff: }}$ :

$$
B_{L}=\lambda_{\text {res }} / \Delta n_{\text {eff }}
$$

By combining Eq. 6 and Eq. 7 with $B_{L}=4 L_{c}$, another expression for loop length is obtained:

$$
L_{c}=\lambda_{\text {res }}^{2} /\left(4 \Delta \lambda_{\text {res }} n_{\text {eff }}\right)
$$

Equating Eq. 5 and Eq. 8 produces a rule between $\Delta \lambda_{\text {res }}$ and integer $C$ :

$$
\Delta \lambda_{\text {res }}=\lambda_{\text {res }} /(4 C+2)
$$

For near-critical-coupled MLRs with low loss, a $\Delta \lambda_{\text {res }}$ of $250 \mathrm{pm}$ would be sufficient to ensure one eigenmode is maximally recirculated while the other is uncoupled. This requires $C=1550$ for $\lambda_{\text {res }}=1550 \mathrm{~nm}$ and $n_{\text {eff }}=1.45$ (fast axis). From Eq. 5, the loop circumference $L_{c}$ is 1.66 $\mathrm{mm}$ and the loop diameter is $0.53 \mathrm{~mm}$. From Eq. 7, the beat length is $6.63 \mathrm{~mm}$, corresponding to a linear birefringence of $\Delta n_{\text {eff }}=2.34 \times 10^{-4}$. In practice, the magnitude of $\Delta n_{\text {eff }}$ typically ranges from $\sim 10^{-6}$ to $\sim 10^{-4}$.

Conclusion: Microfiber loop resonator based current sensors which exhibit linear birefringence can be detrimental to the measurable Faraday rotation and thus reduces the current sensitivity. For MLRs with a sufficiently large birefringence-induced resonance separation, efficient Faraday rotation can arise when the loop circumference is a quarter of the polarization beat length and the roundtrip phase of the eigenmode in the fast axis is equal to $3 \pi / 2$ plus an integer multiple of $2 \pi$. These design rules are important for fabricating MLR based current sensors that can facilitate an efficient build-up of Faraday rotation for multi-roundtrips despite the presence of linear birefringence.

G. Y. Chen, G. Brambilla and T. P. Newson (Optoelectronics Research Centre, University of Southampton, Southampton, SO17 1BJ, United Kingdom)

E-mail: gyc1g09@orc.soton.ac.uk

\section{References}

1. Belal, M., Song, Z., Jung, Y., Brambilla, G., and Newson, T. P., 'Optical fiber microwire current sensor', Opt. Lett., 2010, 35, (18), pp. 3045-3047.

2. Chen, G. Y., Lee, T., Ismaeel, R., Brambilla, G., Newson, T. P., 'Resonantly enhanced Faraday rotation in a microcoil current sensor', IEEE Photon. Technol. Lett., 2012, 24, (10), pp. 860-863.

3. Belal, M., Song, Z., Jung, Y., Brambilla, G., and Newson, T. P., 'An interferometric current sensor based on optical fiber micro wires', Opt. Express, 2010, 18, (19), pp. 19951-19956.

4. Guo, X., Li, Y., Jiang, X., and Tong, L., 'Demonstration of critical coupling in microfiber loops wrapped around a copper rod', Appl. Phys. Lett., 2007, 91, (7), pp. 073512-1-3.

5. Lim, K. S., Harun, S. W., Damanhuri, S. S. A., Jasim, A. A., Tio, C. K., and Ahmad, H., 'Current sensor based on microfiber knot resonator', Sens. Actuat. A-Phys., 2011, 167, (1), pp. 60-62.

6. Sulaiman, A. H., S. W., Aryangar, I., and Ahmad, H., 'DC current sensing capability of microfibre Mach-Zehnder interferometer', Electron. Lett., 2012, 48, (15), pp. 943-945.

7. Caspar, C., and Bachus, E. J., "Fibre-optics micro-ring-resonator with 2mm diameter," Electron. Lett., 1989, 25, (11), pp. 1506-1508. 